\title{
2D-Photothermal super resolution with sparse matrix stacking
}

\author{
J. Lecompagnon ${ }^{1}$, S. Ahmadi ${ }^{1}$, P. D. Hirsch ${ }^{1}$, M. Ziegler ${ }^{1}$ \\ ${ }^{1}$ Bundesanstalt für Materialforschung und -prüfung, 12200 Berlin, Germany, \\ Julien.Lecompagnon@bam.de
}

\begin{abstract}
Summary:
Thermographic super resolution techniques allow the spatial resolution of defects/inhomogeneities below the classical limit, which is governed by the diffusion properties of thermal wave propagation. In this work, we report on the extension of this approach towards a full frame 2D super resolution technique. The approach is based on a repeated spatially structured heating using high power lasers. In a second post-processing step, several measurements are coherently combined using mathematical optimization and taking advantage of the (joint) sparsity of the defects in the sample.
\end{abstract}

Keywords: super resolution, laser thermography, nondestructive testing, laser scanning, photothermal imaging

\section{Introduction}

Photothermal super resolution (SR) is based on a combination of an experimental scanning strategy and a numerical optimization, which has been proven to be superior to standard thermographic methods in the case of one-dimensional linear defects. Due to complexity constraints, laser scanning SR techniques have been mostly limited to evaluation of one-dimensional defect patterns and/or small Regions of Interest (ROI) $[1,2,3]$. Extending the SR problem to more dimensions significantly increases the amount of measurement data and the number of measurements required to achieve sufficient defect resolution to cover large areas. With the incorporation of a limited number of priors, such as a sparse representation of the defect density, and with a purposeful exploitation of the sparse nature of the underlying physical models, the increased complexity can be made manageable.

\section{Methods}

The surface temperature of a thin plate exposed to a heating $Q$ with spatial structure $I_{x, y}$ and temporal structure $I_{t}$ can be described by:

$$
\begin{gathered}
T_{\text {meas }}(x, y, z=0, t)=T_{0}+\Phi_{P S F}(x, y, t) *_{x, y} a(x, y) \\
\Phi_{P S F}(x, y, t)=\frac{2 \cdot Q}{c_{p} \rho(4 \pi \alpha t)^{3 / 2}} \cdot e^{-\frac{(x-\hat{x})^{2}+(y-\hat{y})^{2}}{4 \alpha t}} \\
\cdot \sum_{n=-\infty}^{\infty} R^{2 n+1} e^{-\frac{(2 n d)^{2}}{4 \alpha t}} *_{t} I_{t}(t) \\
a(x, y)=a_{0}(x, y) *_{x, y} I_{x, y}(x, y)
\end{gathered}
$$

where $T_{0}$ denotes the initial system temperature, $\rho$ the mass density, $c_{p}$ the specific heat, $\alpha$ the thermal diffusivity, $(\hat{x}, \hat{y})$ the coordinates of the centroid of the excitation, $R$ the thermal wave reflexion coefficient $(R \approx 1), d$ the plate thickness and $a_{0}$ the heat source distribution. The operators $*_{x, y}, *_{t}$ represent the convolution operator in the indicated dimensions [2].
The spatial and temporal dimensions can be discretized as follows:

$$
\begin{aligned}
x_{i} & =i \cdot \Delta x, \quad y_{j}=j \cdot \Delta y, \quad t_{k}=k \cdot f_{\text {cam }}^{-1} \quad(4,5,6) \\
i & \in\left\{1, \ldots, n_{x}\right\}, \quad j \in\left\{1, \ldots, n_{y}\right\}, \quad k \in\left\{1, \ldots, n_{t}\right\}
\end{aligned}
$$

A series of $m \in\left\{1, \ldots, n_{m}\right\}$ independent measurements can be described by:

$$
T_{\text {meas }}\left[x_{i}, y_{j}, t_{k}, m\right]=T_{0}+T_{\text {diff }}\left[x_{i}, y_{j}, t_{k}, m\right]
$$

with $T_{0}$ representing the initial temperature of the sample at $t=0 \mathrm{~s}$ and $T_{\text {diff }}$ being the differential temperature caused by thermal excitation.

In order to reduce the problem complexity, the time dimension can be eliminated by choosing a timestep $t=t_{\text {eval }}$ and only take the temperature change with respect to $T_{0}$ further into account:

$$
T_{\text {diff }}\left[x_{i}, y_{k}, m\right]=T_{\text {meas }}\left[x, y, t=t_{\text {eval }}, m\right]-T_{0}
$$

The spatial dimensions can then be merged by flattening to a single dimension $r$, applying a bijective transform assigning an index $n \in\left\{1, \ldots, n_{y} \cdot n_{x}\right\}$ to every pixel coordinate $\left[x_{i}, y_{j}\right]$ :

$$
\begin{gathered}
T_{r}[n, m]=T_{\text {diff }}\left[x_{i}, y_{j}, m\right] \\
{[i, j]_{\mathrm{n}}=\left\lfloor\left\lfloor\frac{n-1}{n_{y}}\right\rfloor+1, n-\left\lfloor\frac{n-1}{n_{y}}\right\rfloor n_{y}\right\rfloor}
\end{gathered}
$$

The inverse transform of Eq. (10) can be applied to reshape the data back to a two-dimensional image.

As an approximative model, the defect response can be defined as the convolution of the thermal PSF as a Green's function kernel and the heat source distribution $a_{r}[n, m][4]$ :

$$
\Phi_{P S F, r}[n] *_{n} a_{r}[n, m]=T_{r}[n, m]
$$

The single measurement solutions can then be merged by summation.

$$
a_{r e c}[n]=\sum_{m} a_{r}[n, m]
$$


To efficiently solve Eq. (11), it can be transformed to a multiplicative problem by introducing the discrete convolution matrix $\mathrm{h}\left(\Phi_{P S F}\right)$, such that:

$$
h\left(\Phi_{P S F, r}^{\mathrm{m}}\right) \cdot a_{r}^{m}=\left[\begin{array}{c}
0 \\
T_{r}^{m} \\
0
\end{array}\right]=T_{r 0}^{m}
$$

with dimensions $\quad \Phi_{P S F, r}^{\mathrm{m}} \in \mathbb{R}^{n_{x} \cdot n_{y},} \quad h\left(\Phi_{P S F, r}\right) \in$ $\mathbb{R}^{2 n_{x} \cdot n_{y}-1 \times n_{x} \cdot n_{y}}$ and $T_{r 0}^{m} \in \mathbb{R}^{2 n_{x} \cdot n_{y}-1}$. The convolution matrix $h$ is a sparse lower triangular matrix with Toeplitz-structure, allowing it to be stored memory-efficient despite its large dimensions.

This leads to solving $n_{m}$ multiplicative inversion problems. In order to be able to exploit the joint sparsity between measurements in all $n_{m}$ equations, they need to be solved simultaneously. This can be achieved by stacking:

$$
H \cdot A=\left[\begin{array}{ccc}
h & 0 & 0 \\
0 & \ddots & 0 \\
0 & 0 & h
\end{array}\right] \cdot\left[\begin{array}{c}
a_{r}^{1} \\
\vdots \\
a_{r}^{n_{m}}
\end{array}\right]=\left[\begin{array}{c}
T_{r 0}^{1} \\
\vdots \\
T_{r 0}^{n_{m}}
\end{array}\right]=T_{R 0}
$$

with dimensions $H \in \mathbb{R}^{\left(2 n_{x} \cdot n_{y}-1\right) \cdot n_{m} \times n_{x} \cdot n_{y} \cdot n_{m}}, \quad A \in$ $\mathbb{R}^{n_{x} \cdot n_{y} \cdot n_{m}}$ and $T_{R 0}^{m} \in \mathbb{R}^{\left(2 n_{x} \cdot n_{y}-1\right) \cdot n_{m}} \cdot H$ is a sparse diagonal block matrix, which makes it efficient to store. To enhance sparsity even further, a threshold to $H$ is applied where $H<10^{-6}=0$.

Since all measured data is prone to noise and due to the ill-posed nature of the problem, $H$ can not be inverted easily. Therefore the "Blocksoft" regularization method is applied [5]:

$$
\min _{A} \frac{1}{2}\left\|\mathrm{H} \mathrm{A}-T_{R 0}\right\|_{2}^{2}+\lambda_{21}\|A\|_{2,1}+\lambda_{2}\|A\|_{2}
$$

Where $\|A\|_{2,1}$ denotes the $\mathrm{L}_{2,1}$-norm $\|A\|_{2,1}=$ $\sum_{m} \sqrt{\sum_{n} A_{n}^{m 2}}$ and $\lambda_{21}, \lambda_{2}$ are free regularization parameters. This $L_{2,1}$-norm couples the single measurements to achieve super resolution. Eq. (15) can be solved for $A$ iteratively with the ADMM algorithm [6].

\section{Results}

To test the performance of the proposed algorithm we have examined a purpose made sample with blind structured heating. For this a diode laser with a wavelength of $940 \mathrm{~nm}$ and a total output power of $P_{\text {total }}=$ $500 \mathrm{~W}$ has been utilized while measuring the sample surface temperature with an IR camera ImageIR 9300 with a framerate of $f_{\text {cam }}=100 \mathrm{~Hz}$ and a spatial resolution of $\Delta \mathrm{x}, \Delta y=60 \mu \mathrm{m}$.

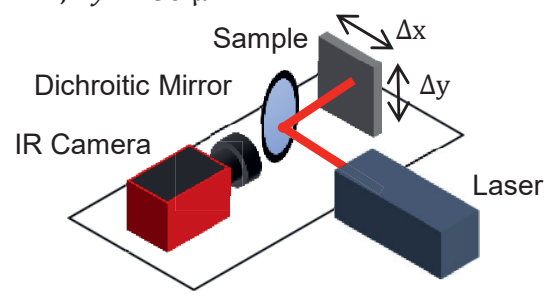

Fig. 1. Schema depicting the experimental setup for active thermography measurements with laser excitation in reflection configuration

The sample under investigation has been additively manufactured from $316 \mathrm{~L}$ stainless steel $\left(\alpha=3.76 \cdot 10^{-6} \frac{\mathrm{m}^{2}}{\mathrm{~s}}, \rho=7950 \frac{\mathrm{kg}}{\mathrm{m}^{3}}, \quad c_{p}=502 \frac{\mathrm{J}}{\mathrm{kg} \mathrm{K}}\right)$ and features several cubical defect pairs $0.5 \mathrm{~mm}$ beneath the front surface. Each defect has an edge length of $2 \mathrm{~mm}$ with decreasing distances within the pairs.

220 blind measurements with randomly sampled excitation positions across the ROI with a laser power of $P=15 \mathrm{~W}$, a pulse duration of $t_{o n}=0.2 \mathrm{~s}$ and a laser spot size of $d_{\text {spot }}=1.5 \mathrm{~mm}$ have been conducted. Applying our previously described 2D-SR evaluation technique, the resulting defect reconstruction $a_{r e c}$ is displayed in Fig. 2.

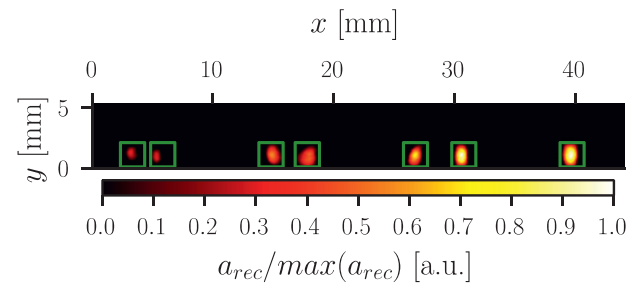

Fig. 2 Resulting defect density $\mathrm{a}_{\text {rec }}\left(x_{i}, y_{j}\right)$ from solving Eq. (15) with an ADMM penalty $\rho_{A D M M}=16, \lambda_{21}=$ $375, \lambda_{2}=20$ for $t_{\text {eval }}=0.3 \mathrm{~s}$. The green boxes indicate the defect position and sizes. All defects and even close defect spacings up to $0.5 \mathrm{~mm}$ are resolved.

For reference, a single measurement with homogenous illumination across the sample surface has been performed. The measured data is shown in Fig. 3.

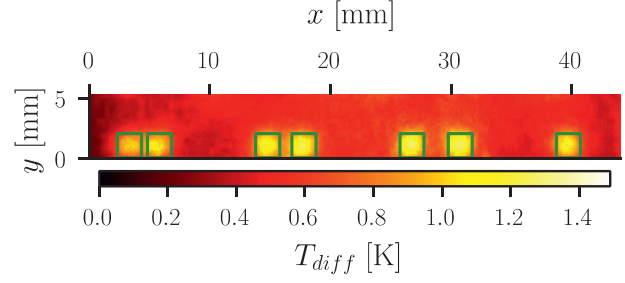

Fig. 3. Thermogram for full area homogeneous illumination for reference. $P=450 \mathrm{~W}, t_{\text {on }}=0.5 \mathrm{~s}$ sampled at $t_{\text {eval }}=0.5 \mathrm{~s}$. All defects are clearly visible but closer defects cannot be resolved independently.

\section{References}

[1] S. Ahmadi, P. Burgholzer et al., Photothermal super resolution imaging: A comparison of different thermographic reconstruction techniques, NDT \& E International,Volume 111 (2020); doi: 10.1016/j.ndteint.2020.102228

[2] S. Ahmadi, P. Burgholzer et al., Super resolution laser line scanning thermography, Optics and Lasers in Engineering, Volume 134 (2020); doi: 10.1016/j.optlaseng.2020.106279

[3] S. Ahmadi, J. Lecompagnon et. al., Laser excited super resolution thermal imaging for nondestructive inspection of internal defects, ArXiv preprint (2020), ArXiv:2007.03341

[4] K. Cole, J.V. Beck et al., Heat conduction using green's functions, second edition. Taylor \& Francis (2010); doi: 10.1201/9781439895214

[5] T. W. Murray, M. Haltmeier et al., Super-resolution photoacoustic microscopy using blind structured illumination, Optica 4, 17-22 (2017); doi: 10.1364/OPTICA.4.000017

[6] S. Boyd, N. Parikh et al., Distributed Optimization and Statistical Learning via the Alternating Direction Method of Multipliers, Foundations and Trends in Machine Learning 3, 1-122 (2011); doi: $10.1561 / 2200000016$ 